\title{
ALDHIAI induces resistance to CHOP in diffuse large B-cell lymphoma through activation of the JAK2/STAT3 pathway
}

This article was published in the following Dove Press journal:

OncoTargets and Therapy

29 August 2016

Number of times this article has been viewed

\author{
Jinqiong Jiang \\ Yiping Liu \\ Youhong Tang \\ Li Li \\ Ruolan Zeng \\ Shan Zeng \\ Meizuo Zhong
}

Department of Oncology, Xiangya Hospital, Central South University,

Changsha, Hunan, People's

Republic of China
Correspondence: Meizuo Zhong Department of Oncology, Xiangya Hospital, Central South University, 87 Xiangya Road, Changsha 410008, Hunan, People's Republic of China Tel +86 I3974829582 Fax +8673 I 89753734 Email zhongmeizuo2015@।26.com
Abstract: Increasing evidence has shown that aldehyde dehydrogenase 1A1 (ALDH1A1), a detoxifying enzyme, is responsible for chemoresistance in a variety of tumors. Although the majority of patients with diffuse large B-cell lymphoma (DLBCL) can be cured with cyclophosphamide, doxorubicin, vincristine, and prednisone (CHOP), chemoresistance is a common cause of treatment failure. This study aims to investigate the significance of ALDH1A1 expression and the mechanism by which ALDH1A1 is involved in the chemoresistance of DLBCL cells. ALDH1A1 expression was assessed in 88 DLBCL tissues by immunohistochemistry. The association between ALDH1A1 expression and outcome was evaluated. We also investigated the effect of ALDH1A1 on CHOP resistance in DLBCL cells using functional analysis. ALDH1A1 expression levels were upregulated in patients with stable or progressive disease after CHOP and its expression positively correlated with expression of STAT3 and p-STAT3. In keeping with these observations, ALDH1A1 expression was significantly associated with short survival of DLBCL patients who received CHOP chemotherapy. In functional assays in Pfeiffer cells, overexpression of ALDH1A1 conferred resistance to CHOP, while silencing of ALDH1A1 using short hairpin RNA had the opposite effect. Furthermore, we also observed that ALDH1A1 could regulate the JAK2/STAT3 pathway, while inhibition of the JAK2/STAT3 pathway by WP1066 negated the effect of ALDH1A1 overexpression. These observations reveal that ALDH1A1 induces resistance to CHOP through activation of the JAK2/STAT3 pathway in DLBCL, and its targeting provides a potential strategic approach for reversing $\mathrm{CHOP}$ resistance.

Keywords: ALDH1A1, diffuse large B-cell lymphoma, CHOP, chemoresistance, JAK2/ STAT3 pathway

\section{Introduction}

Diffuse large B-cell lymphoma (DLBCL) represents the most common subtype of non-Hodgkin lymphoma worldwide, accounting for 30\%-40\% of all newly diagnosed cases. ${ }^{1}$ It is an aggressive disease featuring heterogeneous genetic, phenotypic, and clinical characteristics. The most commonly used initial therapy is an anthracyclinebased combinatorial chemotherapy regimen composed of cyclophosphamide, doxorubicin, vincristine, and prednisone (CHOP). ${ }^{2}$ Even though complete response rates of $40 \%-50 \%$ with chemotherapy can be attained, most patients who do not respond to CHOP will ultimately succumb to their disease. ${ }^{3}$ The major cause of treatment failure is chemotherapeutic drug resistance, which imposes major obstacles to the successful therapy of DLBCL. Although considerable efforts have been made to understand the molecular basis for development of multidrug resistance in DLBCL, current knowledge remains limited. ${ }^{4}$ 
Aldehyde dehydrogenases (ALDHs) are a family of intracellular enzymes responsible for oxidizing aldehydes to carboxylic acids. ${ }^{5}$ ALDH1A1, one of 19 ALDH isoforms, is not only crucial for protecting cells from toxic aldehydes but is also known to play important roles in retinoic acid metabolism, cancer development, and drug resistance. ${ }^{6}$ Mounting evidence has shown that ALDH1A1 can offer cellular protection against many cytotoxic drugs. ${ }^{7}$ This protective role was first observed over 2 decades ago when it was reported that leukemic cells with ALDH activity were highly resistant to cyclophosphamide. ${ }^{8}$ Recently, we have demonstrated that knockdown or inhibition of ALDH1A1 could increase chemosensitivity in DLBCL Farage cells. ${ }^{9}$ However, the role of ALDH1A1 in chemoresistance of DLBCL has not been identified completely. Further research efforts are needed to clarify the exact mechanisms of ALDH1A1 in DLBCL chemoresistance.

JAK/STAT3 signaling pathway is a well-known cell survival signal that contributes to chemoresistance in a variety of cancer cells. ${ }^{10}$ In lymphoid malignancies, a pathogenic role for STAT3 has been shown in multiple myeloma, anaplastic large T-cell lymphoma, Hodgkin lymphoma, and, recently, in activated B-cell-DLBCL. ${ }^{11}$ Moreover, Alas et al demonstrated that chemical inhibitors of the STAT3 pathway are a novel class of compounds capable of reversing drug resistance in lymphoid-derived tumors. ${ }^{12}$ We have previously demonstrated that knockdown or inhibition of ALDH1A1 could decrease STAT3/NF- $\kappa B$ activity. ${ }^{9}$ However, the pathway through which ALDH1A1 induces chemoresistance is not known, and the potential role of pathway inhibitors in reversing chemoresistance has not been investigated.

In the present study, we investigated the role of ALDH1A1 in CHOP resistance using the human DLBCL cell line Pfeiffer transduced with an ALDH1A1-expressing or ALDH1A1-shRNA (short hairpin RNA) lentivirus. Moreover, the correlation between ALDH1A1 expression and clinical drug response was analyzed in DLBCL patients. We also examined the association between ALDH1A1 and the JAK2/STAT3 pathway in relation to CHOP chemoresistance. Taken together, our results support a model in which ALDH1A1 confers resistance to CHOP through the activation of the JAK2/STAT3 pathway, indicating its potential value as a therapeutic target in DLBCL.

\section{Materials and methods}

\section{Reagents and antibodies}

Antibodies against ALDH1A1 and phospho-JAK2 (tyrosine 570, tyrosine 931, and tyrosine 1007) were purchased from Abcam (Cambridge, UK). Antibodies against JAK2, STAT3,
phospho-STAT3 (tyrosine705, serine 727), Bcl-2, and cyclin D1 were obtained from Cell Signaling Technology (Danvers, MA, USA). Cyclophosphamide and prednisone were purchased from Sigma-Aldrich Co. (St Louis, MO, USA) and dissolved according to the manufacturer's instructions. Doxorubicin and vincristine were obtained from KeyGen Biotechnology (Nanjing, People's Republic of China). All CHOP reagents were stored at $-80^{\circ} \mathrm{C}$. The JAK2/STAT3 inhibitor, WP1066, was obtained from Selleckchem (Houston, TX, USA).

\section{Patients and specimens}

Eighty-eight patients treated for DLBCL in the Department of Oncology and Hematology, Xiangya Hospital, Central South University (Changsha, People's Republic of China), between January 2012 and November 2013, were selected for this study. The criteria for study enrollment were as follows: 1) histopathologically diagnosed DLBCL, 2) newly diagnosed and without a history of other tumors, 3) availability of paraffinembedded specimens, and 4) a treatment regime involving at least four cycles of the CHOP regimen as induction therapy. Detailed clinicopathological information of the patients is listed in Table 1. After the fourth cycle of CHOP therapy, the response to chemotherapy was assessed according to the International Working Group standardized response criteria for non-Hodgkin lymphoma. ${ }^{13}$ We defined the high-sensitivity group as patients showing partial or complete response following CHOP and the low-sensitivity group as patients showing stable or progressive disease. Overall survival time was calculated as the period between the date of diagnosis and the date of death or the last follow-up. The study was approved by the Research Ethics Committee of Xiangya Hospital, Central South University, and written informed consent was obtained from all the patients involved in this study.

\section{Cell lines and cultures}

The human DLBCL cell lines Pfeiffer (CRL 2632) and Farage (CRL 2630) were obtained from the American Type Culture Collection (ATCC, Manassas, VA, USA). The cells were cultured in Roswell Park Memorial Institute (RPMI) medium 1640 (Corning Incorporated, Corning, NY, USA), supplemented with 10\% fetal bovine serum (Corning Incorporated) in a humidified atmosphere of $37^{\circ} \mathrm{C}$ at $5 \% \mathrm{CO}_{2}$. Cells were passaged every $2-3$ days to maintain a density of $1-2 \times 10^{6}$ cells $/ \mathrm{mL}$.

\section{Construction of recombinant plasmids and lentivirus production}

ALDH1A1 coding sequence and ALDH1A1 shRNA in a recombinant lentivirus gene delivery system were 
Table I Correlation between ALDHIAI, STAT3, or p-STAT3 expression and clinicopathological parameters in 88 DLBCL patients

\begin{tabular}{|c|c|c|c|c|c|c|c|c|c|c|}
\hline \multirow[t]{2}{*}{ Parameters } & \multirow[t]{2}{*}{$\mathbf{n}$} & \multicolumn{3}{|c|}{ ALDHIAI expression } & \multicolumn{3}{|c|}{ STAT3 expression } & \multicolumn{3}{|c|}{ p-STAT3 expression } \\
\hline & & High & Low & $P$-value & High & Low & $P$-value & High & Low & $P$-value \\
\hline \multicolumn{11}{|l|}{ Age (years) } \\
\hline$<60$ & 70 & 30 & 40 & 0.586 & 50 & 20 & 0.769 & 37 & 33 & 0.139 \\
\hline$\geq 60$ & 18 & 9 & 9 & & 14 & 4 & & 13 & 5 & \\
\hline \multicolumn{11}{|l|}{ Sex } \\
\hline Male & 37 & 17 & 20 & 0.793 & 29 & 8 & 0.311 & 23 & 14 & 0.389 \\
\hline Female & 51 & 22 & 29 & & 35 & 16 & & 27 & 24 & \\
\hline \multicolumn{11}{|l|}{ B symptoms } \\
\hline Positive & 19 & 12 & 7 & 0.062 & 11 & 8 & 0.101 & 13 & 6 & 0.249 \\
\hline Negative & 69 & 27 & 42 & & 53 & 16 & & 37 & 32 & \\
\hline \multicolumn{11}{|l|}{$\mathrm{LDH}$} \\
\hline Elevated & 33 & 19 & 14 & 0.052 & 22 & II & 0.323 & 21 & 12 & 0.317 \\
\hline Normal & 55 & 20 & 35 & & 42 & 13 & & 29 & 26 & \\
\hline \multicolumn{11}{|l|}{$\beta 2-M G$} \\
\hline Elevated & 15 & 10 & 5 & 0.056 & 9 & 6 & 0.224 & 6 & 9 & 0.149 \\
\hline Normal & 73 & 29 & 44 & & 55 & 18 & & 44 & 29 & \\
\hline \multicolumn{11}{|l|}{ CAI 25} \\
\hline Elevated & 29 & 10 & 19 & 0.193 & 18 & II & 0.116 & 13 & 16 & 0.111 \\
\hline Normal & 59 & 29 & 30 & & 46 & 13 & & 37 & 22 & \\
\hline \multicolumn{11}{|c|}{ DLBCL subtypes } \\
\hline $\mathrm{GCB}$ & 40 & 15 & 25 & 0.240 & 25 & 15 & 0.049 & 18 & 22 & $0.04 I$ \\
\hline Non-GCB & 48 & 24 & 24 & & 39 & 9 & & 32 & 16 & \\
\hline \multicolumn{11}{|l|}{ Clinical stage } \\
\hline$I+I I$ & 36 & 11 & 25 & 0.031 & 23 & 13 & 0.121 & 17 & 19 & 0.051 \\
\hline III + IV & 52 & 28 & 24 & & 41 & II & & 33 & 19 & \\
\hline \multicolumn{11}{|l|}{ IPI score } \\
\hline $0-2$ & 63 & 22 & 41 & 0.005 & 43 & 20 & 0.104 & 39 & 24 & 0.126 \\
\hline $3-5$ & 25 & 17 & 8 & & 21 & 4 & & 11 & 14 & \\
\hline
\end{tabular}

Abbreviations: LDH, lactate dehydrogenase; $\beta 2-M G, \beta 2$-microglobulin; CAI25, cancer antigen I25; DLBCL, diffuse large B-cell lymphoma; GCB, germinal center B-cell; IPI, International Prognostic Index; ALDHIAI, aldehyde dehydrogenase IAI; p-STAT3, phosphorylated-STAT3.

constructed by GeneChem Biomedical Co. Ltd (Shanghai, People's Republic of China) for ALDH1A1 overexpression and knockdown. A blank vector lentivirus gene delivery system was used as a negative control. Lentivirus was transfected into Pfeiffer cells at an optimal multiplicity of infection of 100 supplemented with enhanced infection solution and polybrene (GeneChem Biomedical Co. Ltd) according to the manufacturer's recommended protocol. Validation of the knockdown and overexpression of ALDH1A1 was performed at the protein level by Western blotting.

\section{In vitro drug sensitivity assay}

Cells were seeded into 96-well plates at a density of $2 \times 10^{4}$ cells per well in a volume of $100 \mu \mathrm{L}$. CHOP were added at the standard clinically used ratio of $80 / 5.5 / 0.16 / 11.1^{14}$ and concentrations ranging from 40 to $1,280 \mathrm{ng} / \mathrm{mL}$. Cells were treated with various concentrations of CHOP for 48 hours. Control columns contained cells without drug and blank columns contained only the medium. Cell survival was then analyzed via the Cell Counting Kit-8 assay (CCK-8;
7SeaPharmTech, Shanghai, People's Republic of China) according to the manufacturer's instructions. After incubation with $10 \mu \mathrm{L}$ of CCK-8 reagent for 4 hours, the absorbance was measured at a wavelength of $450 \mathrm{~nm}$ by an automatic plate reader. All procedures were performed in triplicate.

\section{Colony formation in soft agar}

For colony formation assay, a bottom layer ( $0.6 \%$ agar) was prepared with RPMI 1640 medium containing 10\% fetal bovine serum. A top layer $(0.3 \%$ agar) was prepared with the same medium as described earlier but containing 1,000 indicated cells per well treated with or without $640 \mathrm{ng} / \mathrm{mL}$ CHOP. Plates were incubated for nearly 14 days at $37^{\circ} \mathrm{C}$ with $5 \% \mathrm{CO}_{2}$ in a humidified incubator until the formed colonies were visible. The visible colonies were then counted and images were captured. All procedures were performed in triplicate.

\section{Apoptosis assay by flow cytometry}

Cells were seeded into six-well plates and treated with or without $640 \mathrm{ng} / \mathrm{mL}$ CHOP for 48 hours. Cells were harvested 
for Annexin V-fluorescein isothiocyanate and propidium iodide staining. The cells were then analyzed by flow cytometry using fluorescence-activated cell-sorting flow cytometer (BD Biosciences, San Jose, CA, USA). All assays were performed independently in triplicate.

\section{Western blotting}

Total protein was extracted from cells using radioimmunoprecipitation assay buffer and quantified using bicinchoninic acid assay. Equal amounts of protein were separated by sodium dodecyl sulfate polyacrylamide gel electrophoresis before being electrotransferred to polyvinylidene fluoride membranes. After the membranes were blocked with $5 \%$ skimmed milk for 1 hour at room temperature, they were incubated with primary antibodies overnight at $4^{\circ} \mathrm{C}$. Glyceraldehyde 3-phosphate dehydrogenase (GAPDH) was used as a protein-loading control. After being washed with a mixture of Tris-buffered saline and 0.1\% Tween 20 (TBST) three times, the membranes were incubated with horseradish peroxidase-conjugated secondary antibody for 1 hour at room temperature. After washing again with TBST, the protein bands were detected by chemiluminescence. Blots shown are representative of a minimum of three separate experiments.

\section{Immunohistochemistry}

The paraffin-embedded sections were cut at $4 \mathrm{~mm}$ thickness and then deparaffinized and rehydrated. After washing in phosphate buffered saline, the slides were blocked for endogenous peroxidase with $3 \%$ hydrogen peroxide in methanol for 10 minutes at room temperature. Sections were then blocked with $10 \%$ goat serum for 10 minutes, followed by incubation with a primary antibody overnight at $4{ }^{\circ} \mathrm{C}$. Monoclonal antibodies used were as follows: ALDH1A1, STAT3, and p-STAT3. After washing, sections were incubated with a biotin-labeled secondary antibody for 10 minutes at room temperature. Then, streptavidin peroxidase reagent was applied for 10 minutes. Coloration was developed by a 4-minute incubation with 3,3'-diaminobenzidine solution and the sections were counterstained with hematoxylin. The immunohistochemistry-stained slides were independently evaluated by two pathologists blinded to the clinical parameters. The extent of stained cells was scored as $0(0 \%-5 \%), 1(6 \%-25 \%), 2(26 \%-50 \%)$, or $3(51 \%-100 \%)$. The staining intensity was scored as 0 (negative), 1 (weak), 2 (medium), or 3 (strong). The sum of the extent and intensity scores was used as the final staining score (0-6). For the statistical analysis, we divided cases into two groups: low expression (with final scores $<3$ points) and high expression (with final scores $\geq 3$ points).

\section{Statistical analysis}

All experiments were run in triplicate, and results are presented as the mean \pm standard deviation. The association between protein expression levels and clinicopathological parameters was analyzed by the chi-square test. Statistical analyses were carried out using either the Student's $t$-test or an analysis of variance. Kaplan-Meier and log-rank methods were used to analyze survival curves. The Cox regression model was used for analysis of prognostic factors. Differences were considered statistically significant when the two-sided $P$-value was less than 0.05. Statistical analyses were performed with the SPSS 17.0 software (SPSS Inc., Chicago, IL, USA).

\section{Results}

\section{The protein expression level of ALDHIAI is upregulated in DLBCL Pfeiffer and Farage cells}

We first analyzed ALDH1A1 expression in two human DLBCL cell lines, Farage and Pfeiffer, by Western blot. The human B-cell was used as a control. Although Pfeiffer showed higher ALDH1A1 expression than Farage, the expression in both these cell lines was significantly higher than that of human B-cells (sixfold and 4.5-fold, respectively, $P<0.05$; Figure 1A). Thus, these results indicate that the protein expression level of ALDH1A1 is upregulated in DLBCL (Pfeiffer and Farage) cells.

\section{ALDHIAI is upregulated in CHOP- resistant $D L B C L$ patients and is associated with STAT3/p-STAT3}

We first determined the level of ALDH1A1 expression in cancer tissues of DLBCL patients by immunohistochemical analysis. ALDH1A1 showed a significantly higher expression in samples from patients in the low-sensitivity group compared with those in the high-sensitivity group (Figure 1B). Next, we assayed the expression levels of STAT3 and p-STAT3 in 88 DLBCL specimens by immunohistochemistry and assessed the correlations between the expression levels of STAT3/p-STAT3 and ALDH1A1. As shown in Table 2 and Figure 1C, the expression levels of ALDH1A1, STAT3, and p-STAT3 in tissues from low-sensitivity group patients were significantly higher than those from patients 
A
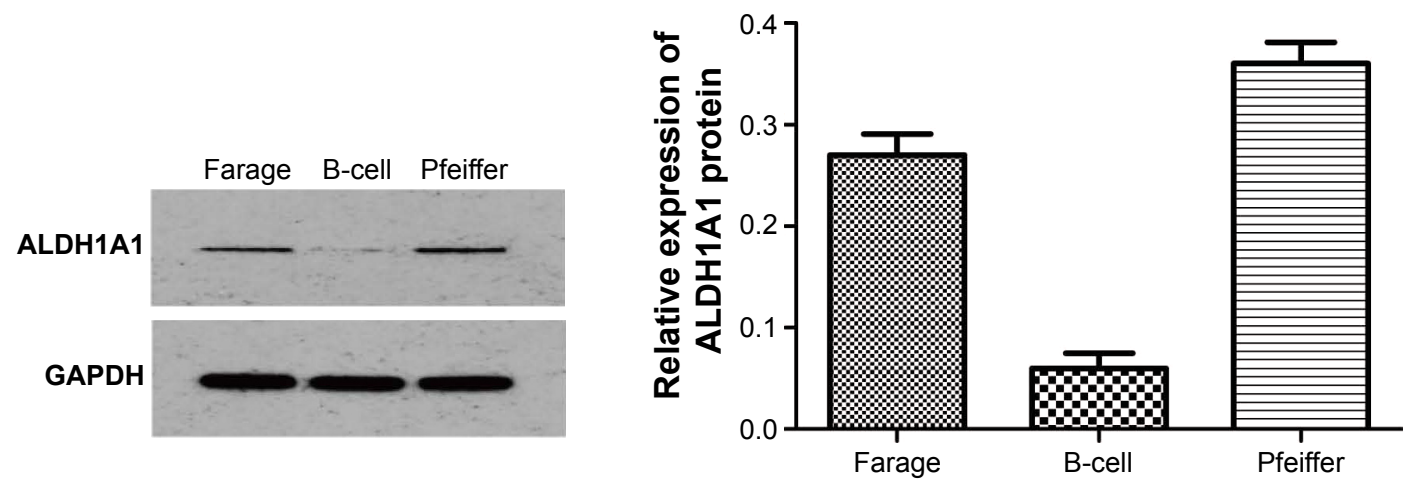

B
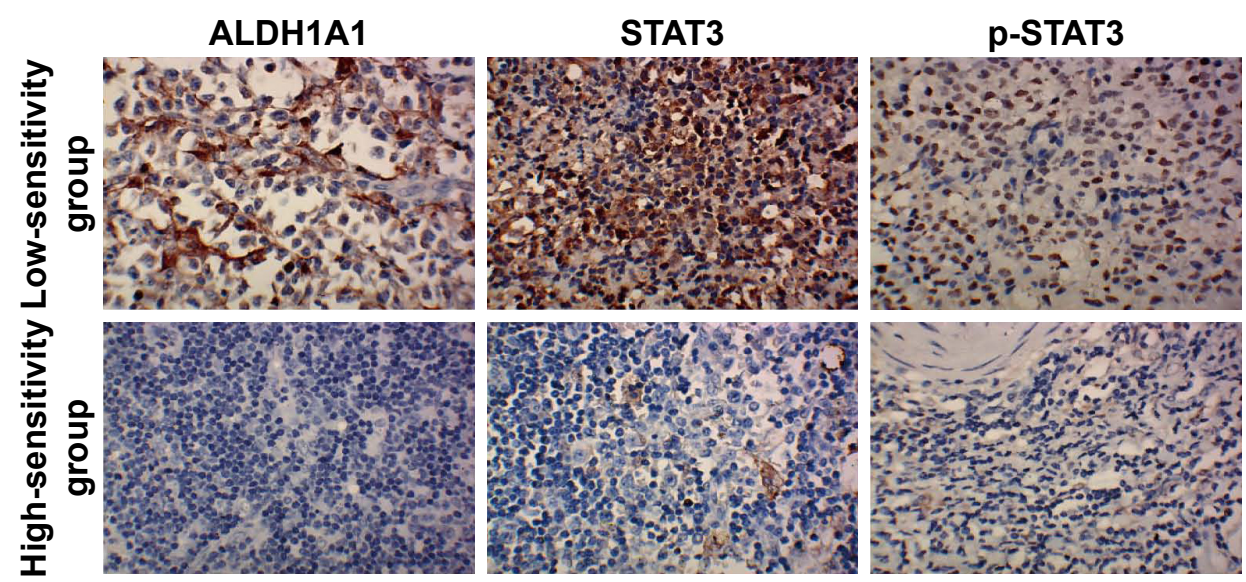

C

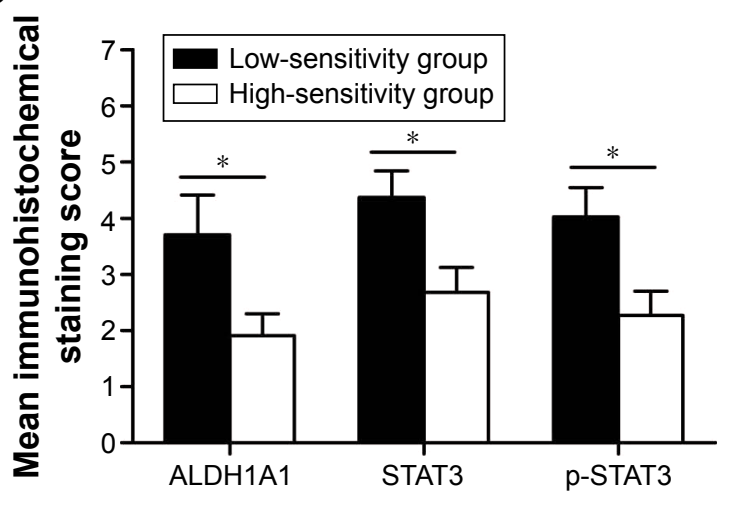

D

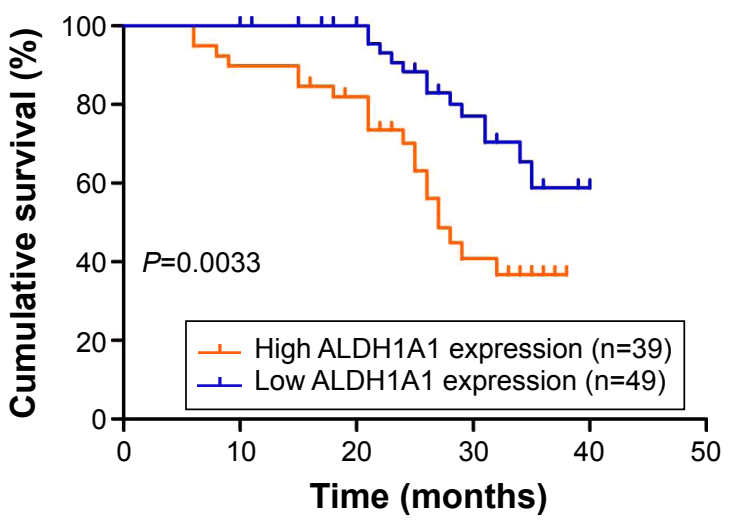

Figure I ALDHIAI expression in DLBCL cell lines and clinical specimens.

Notes: (A) The levels of ALDHIAI protein were quantified using Western blot in two DLBCL cell lines (Farage and Pfeiffer). GAPDH blotting was used as a loading control. Bar graphs are derived from densitometric scanning of the blots. The density of the ALDHIAI blot was normalized against that of GAPDH to obtain a relative blot density. Bars are mean \pm SD from three independent experiments. (B) Representative images of ALDHIAI, STAT3, and p-STAT3 immunohistochemical staining in low sensitivity group and high sensitivity group DLBCL patients (magnification: 200x). (C) Mean immunohistochemiscal staining scores for ALDHIAI, STAT3, and p-STAT3 in low-sensitivity group and high-sensitivity group $D L B C L$ patients. $* P<0.05$. (D) The comparison of overall survival between patients with high ALDHIAI expression and low ALDHIAI expression. Patients with low expression of ALDHIAI had a longer survival than those with high expression (log-rank test, $P=0.0033$ ).

Abbreviations: ALDHIAI, aldehyde dehydrogenase IA I; GAPDH, glyceraldehyde 3-phosphate dehydrogenase; SD, standard deviation; DLBCL, diffuse large B-cell lymphoma; P-STAT3, phosphorylated-STAT3.

in the high-sensitivity group $(P<0.05)$. The correlations between the clinicopathological features of DLBCL patients and expression levels of ALDH1A1, STAT3, and p-STAT3 are summarized in Table 1. There was a close association between the level of ALDH1A1 and clinical stage and
International Prognostic Index. STAT3 and p-STAT3 showed association with DLBCL subtypes. The frequencies of STAT3 and p-STAT3 in the non-germinal center B-cell (GCB)-DLBCL were significantly higher than those in the GCB-DLBCL group $(P<0.05)$. Table 3 shows that the 
Table 2 Immunohistochemical detection of ALDHIAI, STAT3, and p-STAT3 expression in low-sensitivity and high-sensitivity groups of diffuse large B-cell lymphoma patients

\begin{tabular}{|c|c|c|c|c|c|c|c|c|c|}
\hline \multirow[t]{2}{*}{ Group } & \multicolumn{3}{|c|}{ ALDHIAI } & \multicolumn{3}{|l|}{ STAT3 } & \multicolumn{3}{|l|}{ p-STAT3 } \\
\hline & High (\%) & Low & $P$-value & High (\%) & Low & $P$-value & High (\%) & Low & $P$-value \\
\hline Low-sensitivity group & $20(62.5)$ & 12 & 0.009 & $29(90.6)$ & 3 & 0.004 & $28(87.5)$ & 4 & 0.000 \\
\hline High-sensitivity group & $19(33.9)$ & 37 & & $35(62.5)$ & 21 & & $22(39.2)$ & 34 & \\
\hline
\end{tabular}

Abbreviations: ALDHIAI, aldehyde dehydrogenase IAI; P-STAT3, phosphorylated-STAT3.

expression of ALDH1A1 was positively associated with that of STAT3 and p-STAT3 in DLBCL tissues.

\section{High expression of ALDHIAI is correlated with poor outcome in CHOP-treated DLBCL patients}

We conducted Kaplan-Meier survival analysis to study the relationship between ALDH1A1 protein expression and survival. Overall survival was significantly shorter in patients expressing high level of ALDH1A1 (log-rank test; $P=0.0033$; Figure 1D), with a median overall survival of 27 months. In contrast, the median survival could not be determined for patients with low ALDH1A1 expression because many were still alive at the conclusion of the study. We also investigated the influence of ALDH1A1 expression and other clinicopathological parameters on overall survival using multivariate Cox regression analysis. As shown in Table 4, ALDH1A1 expression (hazard ratio [HR] 3.648; 95\% confidence interval [CI] 1.655-8.039; $P<0.05$ ) and International Prognostic Index (HR 2.353; 95\% CI 1.033-5.361; $P<0.05$ ) were independent prognostic factors in DLBCL.

\section{ALDHIAI promotes resistance to CHOP in Pfeiffer cells}

To investigate the role of ALDH1A1 in CHOP resistance in DLBCL cells, we modulated ALDH1A1 expression by transfecting Pfeiffer cells with an ALDH1A1-shRNA vector ("Pfeiffer-shRNA") or an ALDH1A1 expression vector

Table 3 Correlation between ALDHIAI and STAT3, P-STAT3 in DLBCL

\begin{tabular}{lcccc}
\hline \multicolumn{5}{c}{ ALDHIAI expression } \\
\cline { 2 - 5 } & High & Low & $\boldsymbol{r}$ & P-value \\
\hline \multicolumn{5}{c}{ STAT3 } \\
High & 33 & 31 & 0.238 & 0.025 \\
Low & 6 & 18 & & \\
P-STAT3 & expression & & & \\
High & 30 & 20 & 0.362 & 0.001 \\
Low & 9 & 29 & & \\
\hline
\end{tabular}

Abbreviations: ALDHIAI, aldehyde dehydrogenase IAI; DLBCL, diffuse large B-cell lymphoma; P-STAT3, phosphorylated-STAT3.
("Pfeiffer-ALDH1A1"). Cells transfected with empty vector ("Pfeiffer-NC") were used as a control. Forty-eight hours after transfection, ALDH1A1 expression was evaluated by Western blot, which showed that Pfeiffer-ALDH1A1 cells displayed a twofold increase of ALDH1A1 protein, while Pfeiffer-shRNA cells showed a 95.6\% downregulation compared with Pfeiffer-NC cells (Figure 2A).

The half maximal inhibitory concentration (IC50) values were determined to evaluate the sensitivity of Pfeiffer-shRNA cells or Pfeiffer-ALDH1A1 cells to CHOP. It was observed that the silencing of ALDH1A1 expression in Pfeiffer cells led to an increased sensitivity to CHOP (IC50: PfeiffershRNA, 314.48 [272.35-366.06] ng/mL vs Pfeiffer-NC, 645.30 [564.33-750.73] ng/mL). In contrast, overexpression of ALDH1A1 in Pfeiffer cells showed a decreased sensitivity to CHOP (IC50: Pfeiffer-ALDH1A1, 1,122.10 [979.68$1,324.51] \mathrm{ng} / \mathrm{mL}$ vs Pfeiffer-NC, 648.02 [566.30-754.66] $\mathrm{ng} / \mathrm{mL})(P<0.05$; Figure 2B).

Next, to determine the long-term effects of ALDH1A1 on CHOP-inhibited cell proliferation, colony formation assay was performed. As depicted in Figure 2C, when exposed to CHOP treatment $(640 \mathrm{ng} / \mathrm{mL})$, the Pfeiffer-shRNA cells showed decreased colony formation capacity compared with Pfeiffer-NC cells, while ALDH1A1 overexpression had the opposite effect $(P<0.05)$.

Table 4 Multivariate analysis of factors contributing to overall survival in DLBCL patients

\begin{tabular}{lll}
\hline Variables & HR $(95 \% \mathrm{Cl})$ & P-value \\
\hline Age & $0.998(0.972-1.024)$ & 0.852 \\
Sex & $1.095(0.5 I 3-2.335)$ & 0.815 \\
B symptoms & $1.790(0.679-4.72 I)$ & 0.239 \\
LDH & $1.539(0.632-3.748)$ & 0.343 \\
B2-MG & $1.644(0.554-4.883)$ & 0.370 \\
CAI25 & $1.476(0.643-3.390)$ & 0.358 \\
DLBCL subtypes & $0.933(0.4 I I-2.12 I)$ & 0.869 \\
Clinical stage & $1.328(0.592-2.978)$ & 0.491 \\
IPI & $2.353(I .033-5.36 I)$ & 0.042 \\
ALDHIAI & $3.648(I .655-8.039)$ & 0.001 \\
\hline
\end{tabular}

Abbreviations: LDH, lactate dehydrogenase; $\beta 2$-MG, $\beta 2$-microglobulin; CAI 25 , cancer antigen 125; DLBCL, diffuse large B-cell lymphoma; IPI, International Prognostic Index; ALDHIAI, aldehyde dehydrogenase IAI; HR, hazard ratio; $\mathrm{Cl}$, confidence interval. 
A
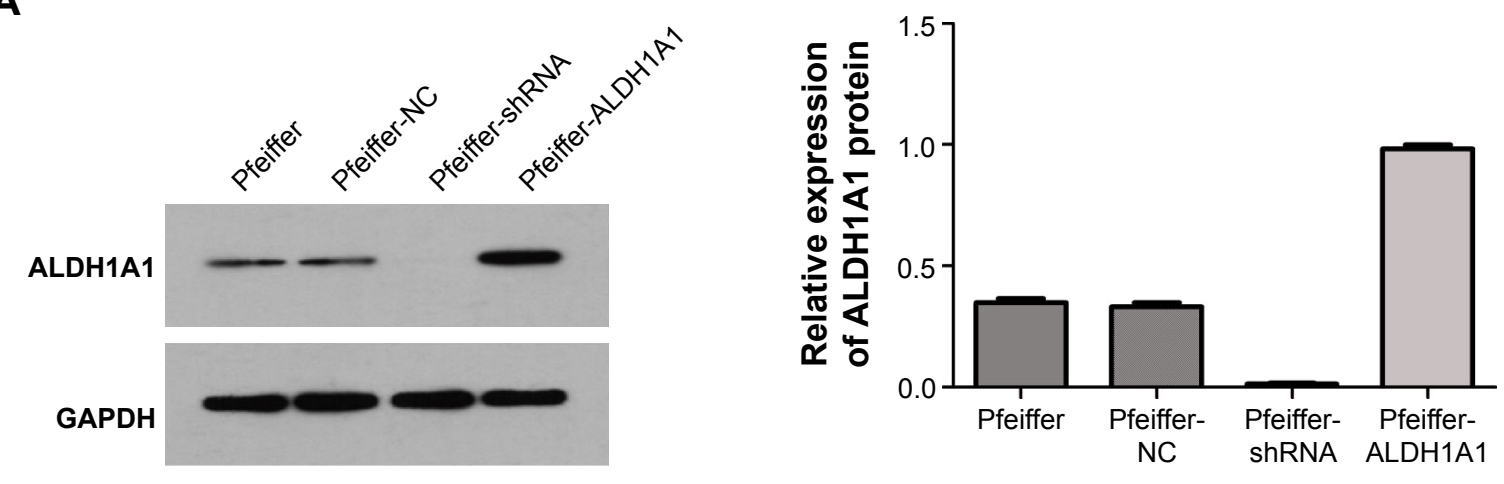

B
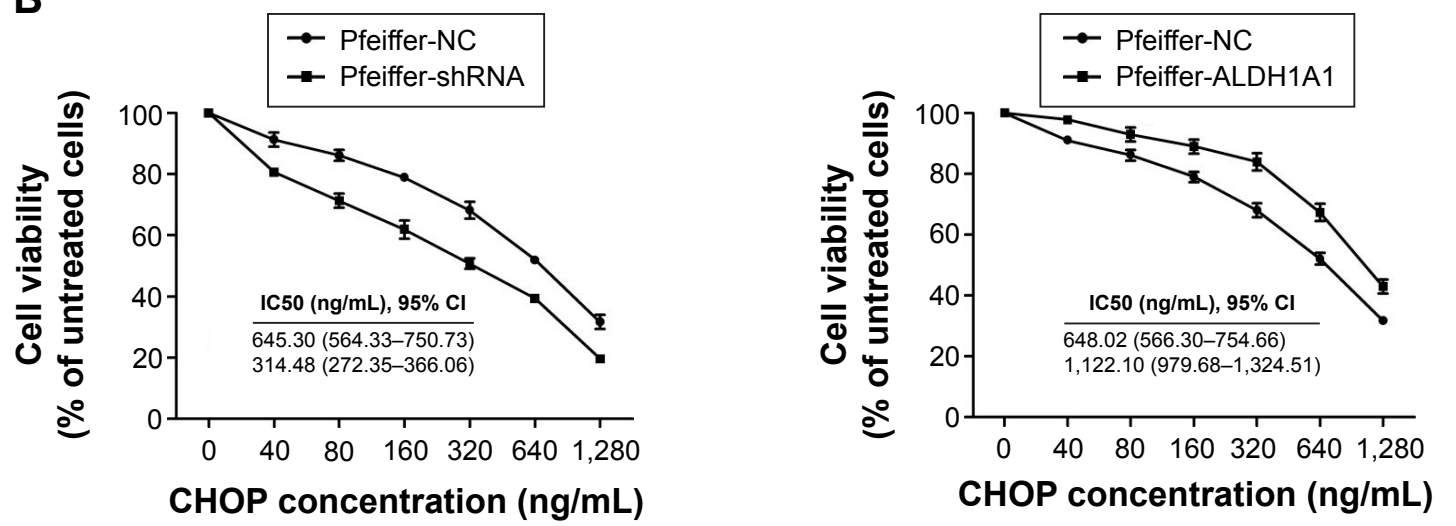

C
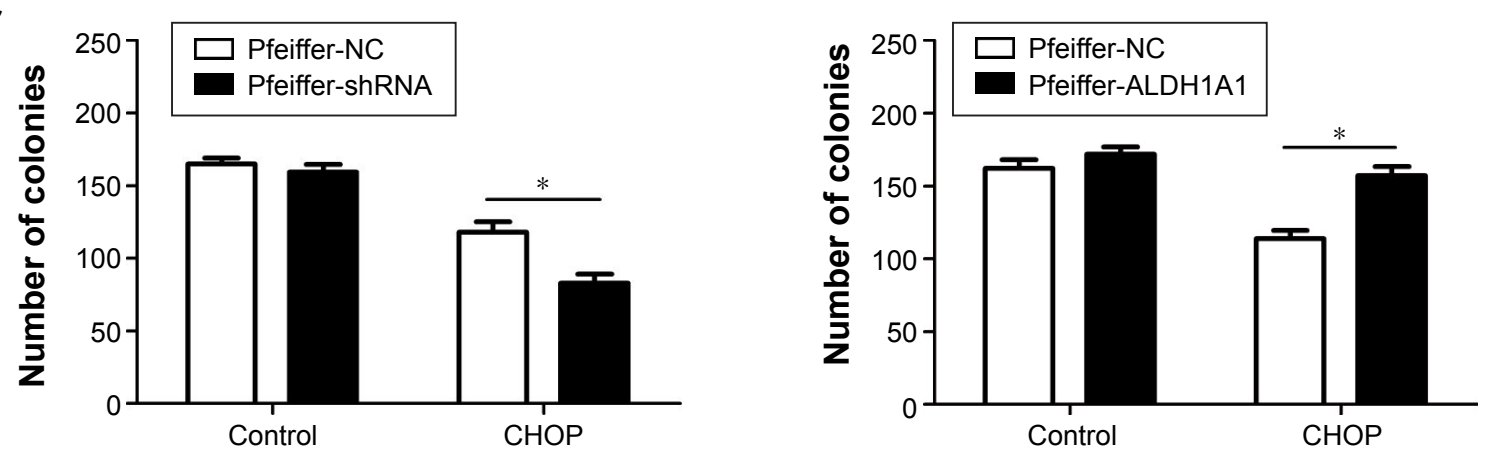

D
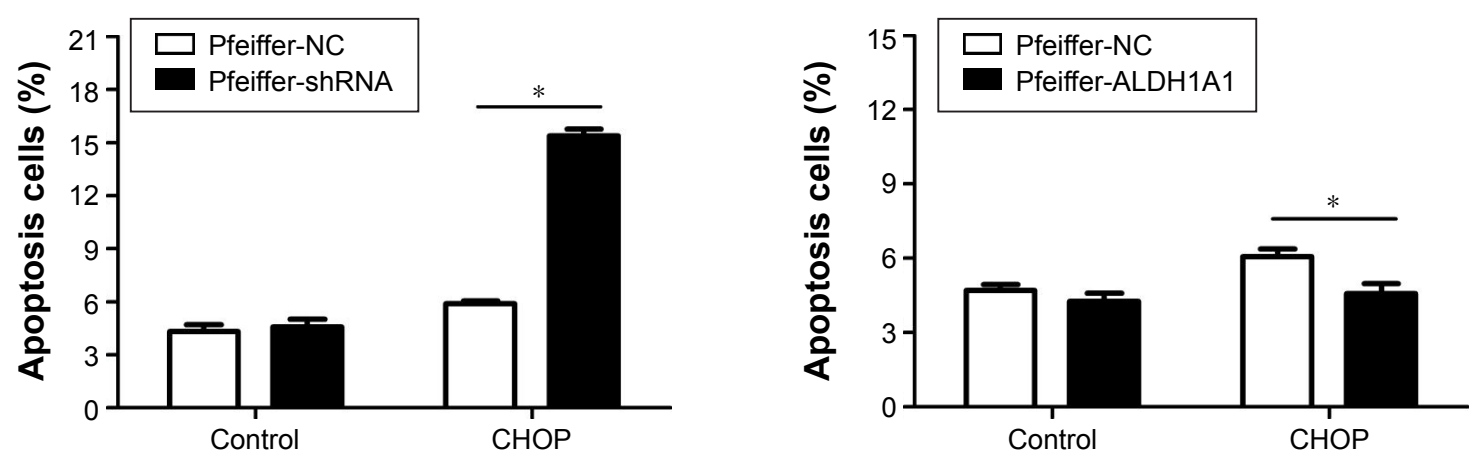

Figure 2 The influence of ALDHIAI on chemoresistance in Pfeiffer cells.

Notes: Cells were transfected with empty vector ("Pfeiffer-NC"), ALDHIAI-shRNA vector ("Pfeiffer-shRNA"), and ALDHIAI expression vector ("Pfeiffer-ALDHIAI"). The blank cells were not transfected ("Pfeiffer"). (A) Expression levels of ALDHIAI and GAPDH proteins were confirmed by Western blotting. (B) The IC50 values of CHOP were determined by CCK-8 assay. Cells were treated with increasing concentrations of CHOP for 48 hours. (C) The long-term effect of ALDHIAI on chemoresistance was studied by colony formation assay. (D) Flow cytometric analysis of apoptosis in cells combined with $\mathrm{CHOP}(0 \mathrm{and} 640 \mathrm{ng} / \mathrm{mL})$. Data are expressed as the mean $\pm S D$ of three individual experiments. $* P<0.05$.

Abbreviations: ALDHIAI, aldehyde dehydrogenase IAI; CHOP, combinatorial cyclophosphamide, doxorubicin, vincristine, and prednisone; GAPDH, glyceraldehyde 3-phosphate dehydrogenase; SD, standard deviation; CCK-8, Cell Counting Kit-8; shRNA, short hairpin RNA; Cl, confidence interval; IC50, half maximal inhibitory concentration. 
To further investigate the effect of ALDH1A1 on cell apoptosis induced by $\mathrm{CHOP}$, flow cytometry analysis was performed. Figure 2D shows that the silencing of ALDH1A1 expression resulted in increased CHOP-induced apoptosis compared with the Pfeiffer-NC group, while ALDH1A1 overexpression in Pfeiffer cells prevented the drug-mediated apoptosis $(P<0.05)$.

\section{ALDHIAI regulates the JAK2/STAT3 pathway in Pfeiffer cells}

The JAK2/STAT3 signaling pathway is implicated in diverse biological processes, including drug resistance. ${ }^{10}$ We performed Western blot to measure whether ALDH1A1
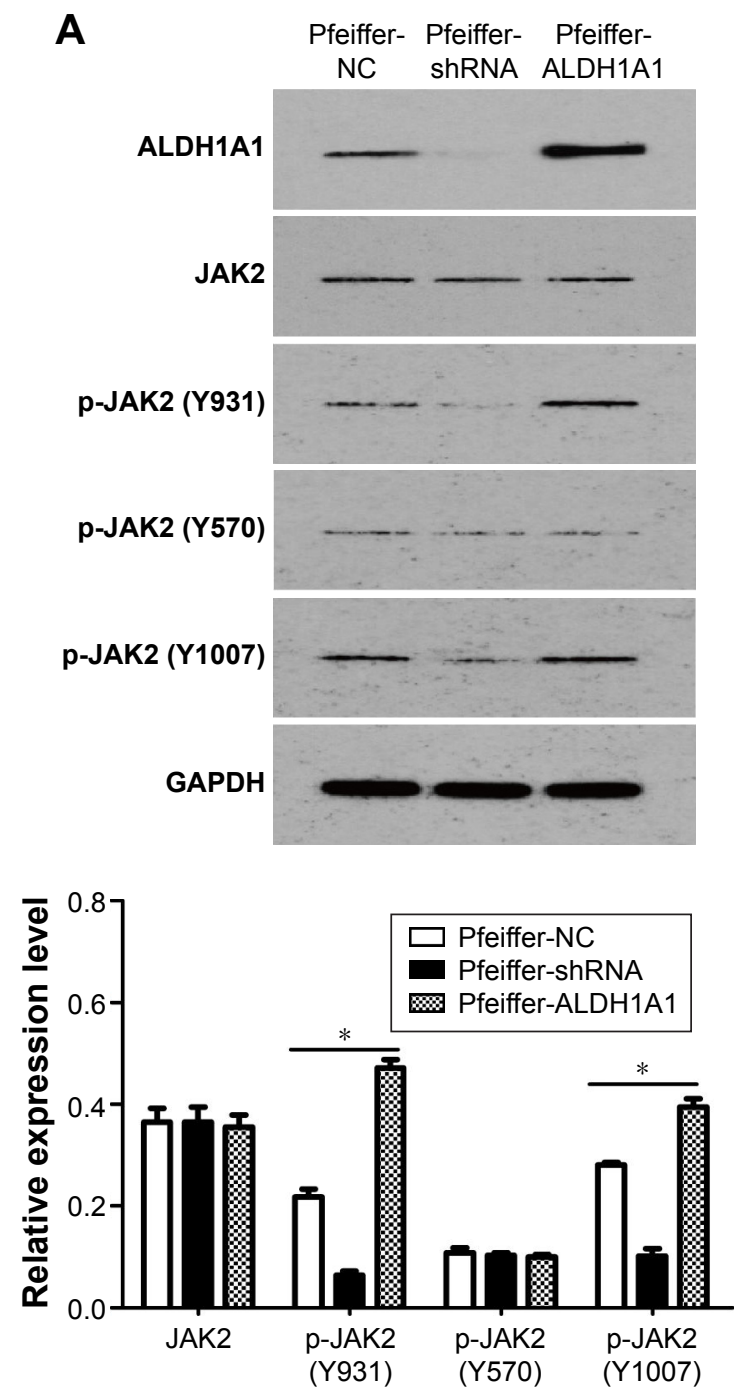

regulated the JAK2/STAT3 pathway in Pfeiffer cells. First, we hypothesized that ALDH1A1 may regulate the expression of JAK2, an upstream activator of STAT3. Western blotting showed that ALDH1A1 overexpression and silencing could increase and decrease the expressions of p-JAK2 (Y931, Y1007), respectively. In contrast, no impact of ALDH1A1 on the expression of JAK2 and p-JAK2 (Y570) was observed (Figure 3A). Then, we investigated whether ALDH1A1 could regulate STAT3 and p-STAT3 in DLBCL. As shown in Figure 3B, overexpression of ALDH1A1 significantly increased the protein levels of STAT3 and p-STAT3 (Y705, S727), while ALDH1A1 silencing significantly reduced the protein levels of these targets. Overall, these results indicate
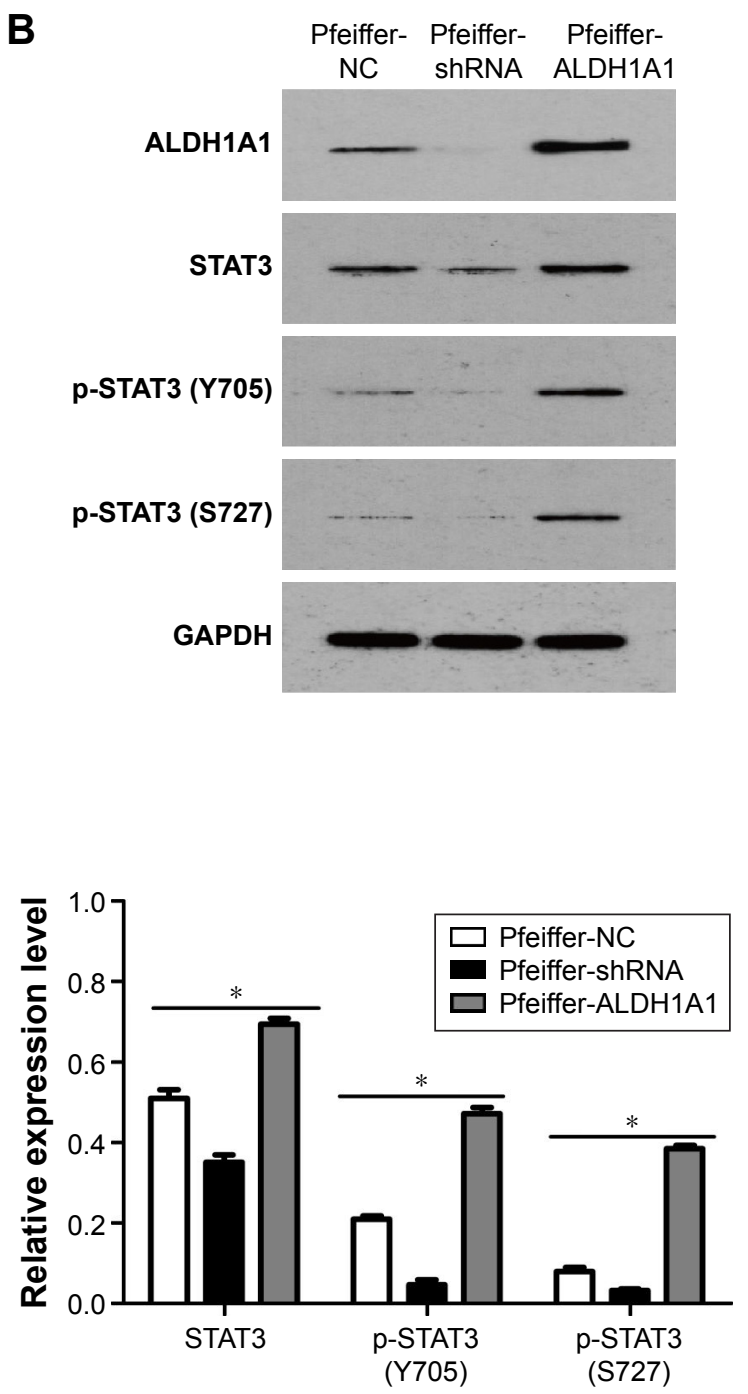

Figure 3 ALDHIAI regulates the JAK2/STAT3 pathway in Pfeiffer cells.

Notes: (A) Effects of ALDHIAI on the expressions of JAK2 and P-JAK2 (Y570, Y93I, and Y1007) were analyzed by Western blotting. (B) Expression levels of STAT3 and P-STAT3 (Y705, S727) were analyzed by Western blotting. GAPDH blotting was used as a loading control. Bar graphs are derived from densitometric scanning of the blots. The density of the ALDHIAI, JAK2/P-JAK2, and STAT3/P-STAT3 blots was normalized against that of GAPDH to obtain a relative blot density. Bars are mean \pm SD from three independent experiments. $* P<0.05$.

Abbreviations: ALDHIAI, aldehyde dehydrogenase IAI; SD, standard deviation; GAPDH, glyceraldehyde 3-phosphate dehydrogenase; shRNA, short hairpin RNA; Pfeiffer-NC, cells transfected with empty vector; Pfeiffer-shRNA, ALDHIAI-shRNA vector; Pfeiffer-ALDHIAI, ALDHIAI expression vector; p-STAT3, phosphorylatedSTAT3; P-JAK2, phosphorylated-JAK2. 
that ALDH1A1 might be involved in activating the JAK2/ STAT3 pathway in DLBCL cells.

\section{ALDHIAI mediates CHOP resistance through the JAK2/STAT3 pathway}

In the previous experiments, we found that the expression of ALDH1A1 was positively correlated with that of STAT3 and p-STAT3 in DLBCL tissues. Moreover, ALDH1A1 could regulate the JAK2/STAT3 pathway in Pfeiffer cells. To further explore whether ALDH1A1 mediates CHOP resistance through the JAK2/STAT3 pathway, we used JAK2/STAT3 signaling-specific inhibitor WP1066. The treatment concentration of WP1066 was $2 \mu \mathrm{mol} / \mathrm{L}$. Western blotting showed that the expression levels of p-JAK2, STAT3, p-STAT3, Bcl-2, and cyclin D1 were increased in ALDH1A1-overexpressing cells, while WP1066 neutralized the effect of ALDH1A1 overexpression (Figure 4A).

Furthermore, we evaluated IC50, colony formation capacity, and cell apoptosis in Pfeiffer-ALDH1A1 cells treated with WP1066. As shown in Figure 4B, WP1066 could increase sensitivity of Pfeiffer-NC cells to CHOP (IC50: Pfeiffer-NC, 644.61 [514.92-851.01] ng/mL vs Pfeiffer-NC+WP1066, 339.64 [270.22-437.68] ng/mL). Moreover, ALDH1A1induced resistance to $\mathrm{CHOP}$, according to IC50, was markedly inhibited by WP1066 (Pfeiffer-ALDH1A1: 1,111.28 [881.06-1,513.95] ng/mL, Pfeiffer-ALDH1A1+WP1066: 504.894 [439.335-589.474] ng/mL; $P<0.05$ ). Colony formation assay was also performed to determine the long-term effects of WP1066. As shown in Figure 4C, WP1066 attenuated ALDH1A1-induced colony formation in Pfeiffer-ALDH1A1 cells exposed to CHOP. Upregulating ALDH1A1 expression in Pfeiffer cells prevented drug-induced apoptosis while WP1066 impaired these effects (Figure 4D).

\section{Discussion}

Despite initial excellent response rates to standard treatment, recurrent disease is still the major cause of mortality in DLBCL. ${ }^{15,16}$ The resistance of lymphoma cells to CHOP has been one of the major challenges in the clinical treatment of DLBCL. Therefore, it is of paramount importance to explore the molecular mechanisms involved in chemoresistance in this disease to identify novel therapeutic targets and develop effective treatment strategies.

ALDH1A1 is the predominant isoform of a family of cytosolic enzymes that catalyze aldehydes to corresponding carboxylic acids, detoxifying many of the free oxygen radicals generated by chemotherapeutic agents. ${ }^{17,18}$ Overexpression of
ALDH1A1 in many drug-resistant cancer cell lines and tissues derived from chemotherapy-resistant patients indicates its potential role in chemoresistance. ${ }^{19}$ Herein, we observed that expression of ALDH1A1 was significantly higher in low-sensitivity DLBCL tissues when compared with their high-sensitivity counterparts. In line with this observation, we found that ALDH1A1 is significantly upregulated in DLBCL cell lines compared with human B-cells. In addition to conferring resistance to cyclophosphamide, accumulating evidence suggests that ALDH1A1 may also be capable of mediating resistance to many other agents such as epirubicin, paclitaxel, cisplatin, and tyrosine kinase inhibitors. ${ }^{20-22}$ ALDH1A1 expression can also identify tumorigenic cells in both solid tumors and B-cell malignancies multiple myeloma and classic Hodgkin lymphoma. ${ }^{17,23}$

In the present study, we confirmed the function of ALDH1A1 on CHOP resistance in Pfeiffer cells by modulation of ALDH1A1 expression using lentivirus transfection. We found that overexpression of ALDH1A1 increased resistance to CHOP. In contrast, ALDH1A1 silencing with shRNA enhanced CHOP-induced cytotoxicity. Taken together, these findings indicate that ALDH1 A1 plays a significant role in CHOP resistance. Importantly, disulfiram, a potent inhibitor of ALDH1A1, has entered a Phase II clinical trial for newly diagnosed glioblastoma (ClinicalTrials.gov identifier NCT01777919). Hence, investigation of the efficacy of targeting ALDH1A1 in DLBCL may be clinically feasible in the near future.

It is generally accepted that JAK2/STAT3 is a critical survival signal, which is involved in cancer development and chemoresistance because of its role in enhancing the expression of survival proteins and preventing cell cycle arrest. ${ }^{10}$ In view of its central role, we investigated whether JAK2/STAT3 is related to the ALDH1A1 regulation of CHOP sensitivity in DLBCL cells. In the current study, we found that expression of ALDH1A1 was positively correlated with that of STAT3 and p-STAT3 in the cancer tissues obtained from DLBCL patients. Moreover, upregulation and silencing of ALDH1A1 increased and decreased the p-JAK2 and p-STAT3 expression in Pfeiffer cells, respectively, suggesting that ALDH1A1 activates JAK2/STAT3 signaling. In addition, we found that treatment of ALDH1A1-Pfeiffer cells with the JAK2/STAT3 inhibitor WP1066 resumed the CHOP cytotoxicity suppressed by ALDH1A1 overexpression. Taken together, our findings may provide new mechanistic insights for CHOP chemoresistance in supporting a model in which ALDH1A1 enhances CHOP resistance through the JAK2/STAT3 pathway. 
A
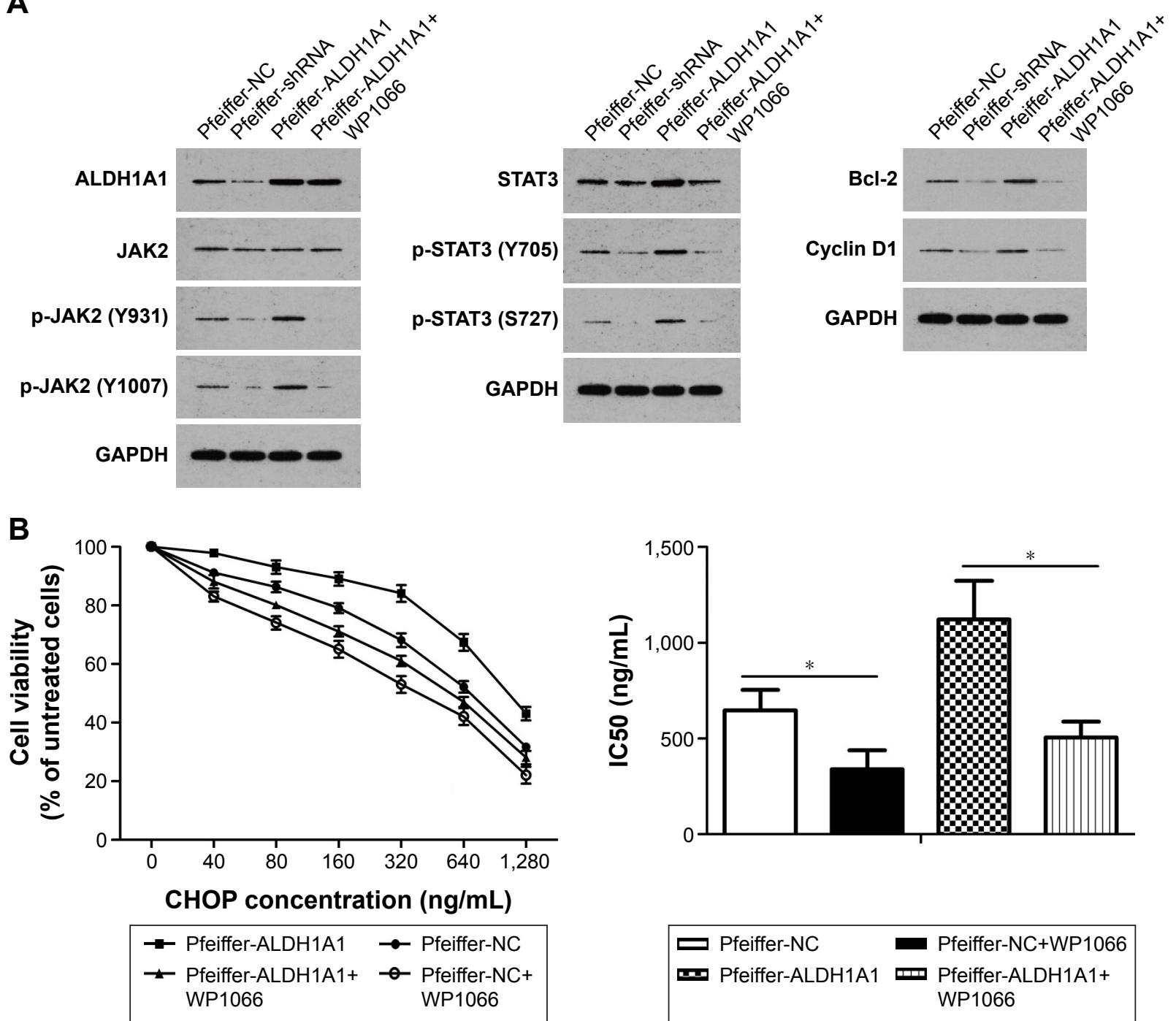

C
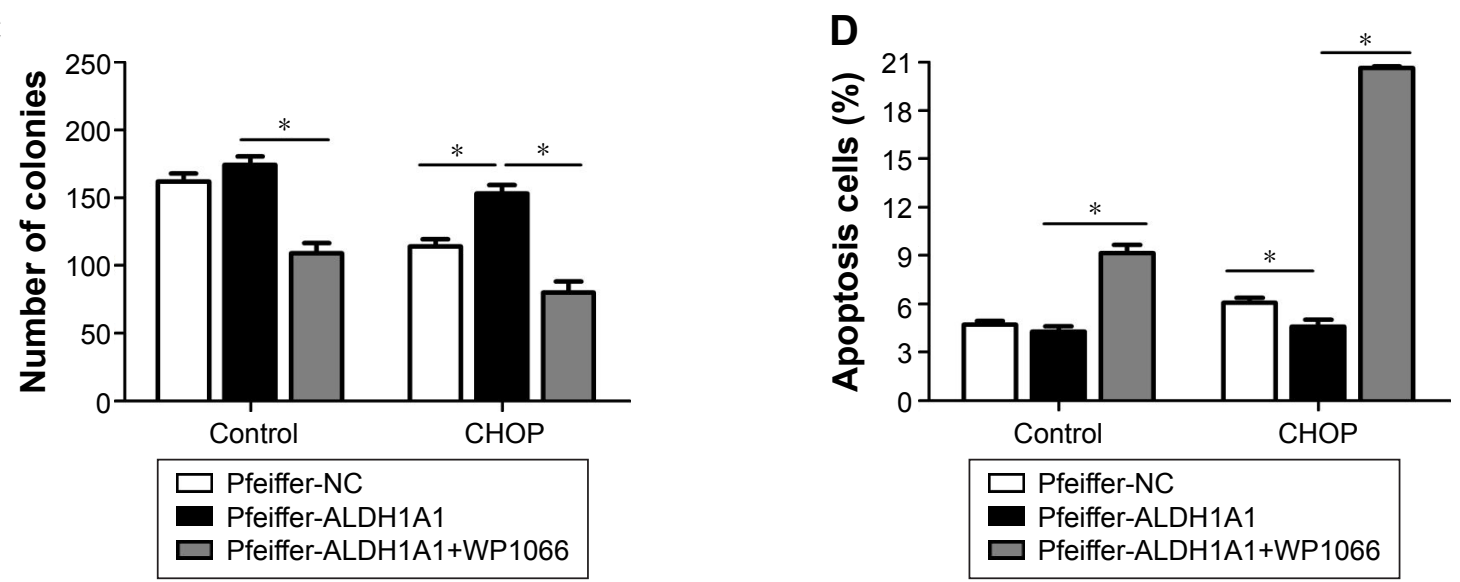

Figure 4 Effects of WPI066 on the JAK2/STAT3 pathway and CHOP resistance induced by ALDHIAI overexpression.

Notes: (A) The protein levels of ALDHIAI, JAK2/p-JAK2, STAT3/p-STAT3, and pathway targets (Bcl-2, cyclin DI) were assayed by Western blotting. GAPDH was used as an internal control. (B) The IC50 values of CHOP were determined by CCK8 assay. (C) Colony formation assay was performed to determine the long-term effects of WPI066. (D) Flow cytometric analysis of apoptosis in cells combined with CHOP (0 and $640 \mathrm{ng} / \mathrm{mL})$. Data are expressed as the mean \pm SD of three individual experiments. $* P<0.05$.

Abbreviations: ALDHIAI, aldehyde dehydrogenase IAI; SD, standard deviation; GAPDH, glyceraldehyde 3-phosphate dehydrogenase; CCK-8, Cell Counting Kit-8; CHOP, combinatorial cyclophosphamide, doxorubicin, vincristine, and prednisone; shRNA, short hairpin RNA; Pfeiffer-NC, cells transfected with empty vector; PfeiffershRNA, ALDHIAI-shRNA vector; Pfeiffer-ALDHIAI, ALDHIAI expression vector; Pfeiffer-ALDHIAI+WPI066, Pfeiffer-ALDHIAI cells treated with WPI066; P-STAT3, phosphorylated-STAT3; P-JAK2, phosphorylated-JAK2; IC50, half maximal inhibitory concentration. 
CHOP chemotherapy induces cell death and cell cycle arrest. ${ }^{24}$ Here, we found that upregulation of ALDH1A1 decreased annexin V-positive apoptotic cells induced by CHOP. Our study also showed that the antiapoptotic protein Bcl-2, which is a downstream effector of STAT3, was increased dramatically after overexpression of ALDH1A1. In a wide range of tumor cells, STAT3 has been shown to induce the expression of $\mathrm{Bcl}-2$, the prosurvival protein that prevents cytochrome c release and inhibits mitochondriadependent cell death. ${ }^{25}$ In addition to inhibition of the intrinsic apoptotic pathway, STAT3 binds directly to the FAS promoter in association with JUN to suppress the transcription of the death receptor, thus leading to inhibition of the extrinsic apoptotic pathway. ${ }^{26}$ Alas et al reported that inhibition of STAT3 activity by AG490 sensitizes resistant nonHodgkin's lymphoma to chemotherapeutic drug-mediated apoptosis. ${ }^{12}$ In agreement with this observation, we found that the JAK2/STAT3 inhibitor WP1066 also promoted apoptosis induced by CHOP. Moreover, we found that overexpression of ALDH1A1 increased the expression level of cyclin D1, which is another downstream target gene of STAT3. In several cell lines, STAT3 and cyclin D1 associate to form a transcriptional repressor complex that binds to the promoter of the cell cycle inhibitor p21WAF1 to downregulate its expression, thus resulting in inhibition of cell cycle arrest. ${ }^{10}$ Together, these observations suggest that the role of JAK2/STAT3 in ALDH1A1-mediated chemoresistance may be dependent on the inhibition of cell cycle arrest and cell apoptosis.

\section{Conclusion}

The effects of ALDH1A1 on CHOP chemosensitivity in vivo and the applicability of the present findings to other DLBCL cell lines remain to be determined. Notwithstanding these limitations, our study provides a better understanding on both the functional role and molecular mechanism of ALDH1A1 in CHOP chemoresistance of DLBCL cells. Our current work reveals that ALDH1A1 confers resistance to $\mathrm{CHOP}$ through activation of the JAK2/STAT3 pathway, indicating its potential value as a therapeutic target in reversing $\mathrm{CHOP}$ resistance.

\section{Acknowledgment}

This study was supported by the National Natural Science Foundation of China (Grant number 81570200).

\section{Disclosure}

The authors report no conflicts of interest in this work.

\section{References}

1. Sehn LH, Gascoyne RD. Diffuse large B-cell lymphoma: optimizing outcome in the context of clinical and biologic heterogeneity. Blood. 2015;125(1):22-32.

2. Liu Y, Zeng L, Zhang S, et al. Identification of differentially expressed proteins in chemotherapy-sensitive and chemotherapy-resistant diffuse large B cell lymphoma by proteomic methods. Med Oncol. 2013;30(2):528.

3. Maxwell SA, Mousavi-Fard S. Non-Hodgkin's B-cell lymphoma: advances in molecular strategies targeting drug resistance. Exp Biol Med. 2013;238(9):971-990.

4. Wilson WH. Drug resistance in diffuse large B-cell lymphoma. Semin Hematol. 2006;43(4):230-239.

5. Duong HQ, Hwang JS, Kim HJ, Kang HJ, Seong YS, Bae I. Aldehyde dehydrogenase $1 \mathrm{~A} 1$ confers intrinsic and acquired resistance to gemcitabine in human pancreatic adenocarcinoma MIA PaCa-2 cells. Int $J$ Oncol. 2012;41(3):855-861.

6. Koppaka V, Thompson DC, Chen Y, et al. Aldehyde dehydrogenase inhibitors: a comprehensive review of the pharmacology, mechanism of action, substrate specificity, and clinical application. Pharmacol Rev. 2012;64(3):520-539.

7. Januchowski R, Wojtowicz K, Zabel M. The role of aldehyde dehydrogenase (ALDH) in cancer drug resistance. Biomed Pharmacother. 2013;67(7):669-680.

8. Hilton J. Role of aldehyde dehydrogenase in cyclophosphamideresistant L1210 leukemia. Cancer Res. 1984;44(11):5156-5160.

9. Song YH, Zhong MZ, Gan PP, et al. ALDH1A1 mediates resistance of diffuse large B cell lymphoma to the CHOP regimen. Tumour Biol. 2014;35(12):11809-11817.

10. Barre B, Vigneron A, Perkins N, Roninson IB, Gamelin E, Coqueret O. The STAT3 oncogene as a predictive marker of drug resistance. Trends Mol Med. 2007;13(1):4-11.

11. Huang X, Meng B, Iqbal J, et al. Activation of the STAT3 signaling pathway is associated with poor survival in diffuse large B-cell lymphoma treated with R-CHOP. J Clin Oncol. 2013;31(36):4520-4528.

12. Alas S, Bonavida B. Inhibition of constitutive STAT3 activity sensitizes resistant non-Hodgkin's lymphoma and multiple myeloma to chemotherapeutic drug-mediated apoptosis. Clin Cancer Res. 2003;9(1): 316-326.

13. Cheson BD, Horning SJ, Coiffier B, et al. Report of an international workshop to standardize response criteria for non-Hodgkin's lymphomas. J Clin Oncol. 1999;17(4):1244.

14. Maxwell SA, Li Z, Jaye D, Ballard S, Ferrell J, Fu H. 14-3-3zeta mediates resistance of diffuse large B cell lymphoma to an anthracycline-based chemotherapeutic regimen. J Biol Chem. 2009;284(33): 22379-22389.

15. Coiffier B, Thieblemont C, Van Den Neste E, et al. Long-term outcome of patients in the LNH-98.5 trial, the first randomized study comparing rituximab-CHOP to standard CHOP chemotherapy in DLBCL patients: a study by the Groupe d'Etudes des Lymphomes de l'Adulte. Blood. 2010;116(12):2040-2045.

16. Karlin L, Coiffier B. Improving survival and preventing recurrence of diffuse large B-cell lymphoma in younger patients: current strategies and future directions. Onco Targets Ther. 2013;6:289-296.

17. Marcato P, Dean CA, Giacomantonio CA, Lee PWK. Aldehyde dehydrogenase: Its role as a cancer stem cell marker comes down to the specific isoform. Cell Cycle. 2014;10(9):1378-1384.

18. Shimada Y, Saji H, Nomura M, et al. Cancer stem cell-related marker expression in lung adenocarcinoma and relevance of histologic subtypes based on IASLC/ATS/ERS classification. Onco Targets Ther. 2013; 6:1597-1604.

19. Czerwinska P, Kaminska B. Regulation of breast cancer stem cell features. Contemporary Oncol. 2015;19(1A):A7-A15.

20. Huang CP, Tsai MF, Chang TH, et al. ALDH-positive lung cancer stem cells confer resistance to epidermal growth factor receptor tyrosine kinase inhibitors. Cancer Lett. 2013;328(1):144-151. 
21. Tanei T, Morimoto K, Shimazu K, et al. Association of breast cancer stem cells identified by aldehyde dehydrogenase 1 expression with resistance to sequential Paclitaxel and epirubicin-based chemotherapy for breast cancers. Clin Cancer Res. 2009;15(12):4234-4241.

22. Schafer A, Teufel J, Ringel F, et al. Aldehyde dehydrogenase 1A1 - a new mediator of resistance to temozolomide in glioblastoma. Neuro-oncology. 2012;14(12):1452-1464.

23. Jones RJ, Gocke CD, Kasamon YL, et al. Circulating clonotypic B cells in classic Hodgkin lymphoma. Blood. 2009;113(23):5920-5926.
24. Pavan A, Spina M, Canzonieri V, Sansonno S, Toffoli G, De Re V. Recent prognostic factors in diffuse large B-cell lymphoma indicate NF-kappaB pathway as a target for new therapeutic strategies. Leuk Lymphoma. 2008;49(11):2048-2058.

25. Bromberg JF, Wrzeszczynska MH, Devgan G, et al. Stat3 as an oncogene. Cell. 1999;98(3):295-303.

26. Ivanov VN, Bhoumik A, Krasilnikov M, et al. Cooperation between STAT3 and c-Jun suppresses Fas transcription. Mol Cell. 2001;7(3): $517-528$.

\section{Publish your work in this journal}

OncoTargets and Therapy is an international, peer-reviewed, open access journal focusing on the pathological basis of all cancers, potential targets for therapy and treatment protocols employed to improve the management of cancer patients. The journal also focuses on the impact of management programs and new therapeutic agents and protocols on

\section{Dovepress}

patient perspectives such as quality of life, adherence and satisfaction. The manuscript management system is completely online and includes a very quick and fair peer-review system, which is all easy to use. Visit http://www.dovepress.com/testimonials.php to read real quotes from published authors. 